\title{
Enriquecimento ambiental como medida para o bem-estar positivo de suínos(Revisão)
}

Environmental enrichment as positive welfare of pigs: a review

\author{
Ana Paula de Assis Maia'; Juliana Sarubbi²; Brenda Batista Lemos Medeiros³; \\ Daniella Jorge de Moura ${ }^{4}$
}

\author{
'Zootecnista, MSc. Doutoranda da Faculdade de Engenharia Agrícola, FEAGRI/UNICAMP, Campinas, SP, Brasil. \\ 2 Médica Veterinária, DSc., Professora Adjunta do Departamento de Zootecnia e Ciências Biológicas \\ (CESNORS/UFSM), Palmeira das Missões, RS, Brasil. \\ ${ }^{3}$ Zootecnista, MSc. Doutoranda da Faculdade de Engenharia Agrícola, FEAGRI/UNICAMP, Campinas, SP, Brasil. \\ ${ }^{4}$ Eng. Agrônoma, DSc., Professora doutorada da Faculdade de Engenharia Agrícola, FEAGRI/UNICAMP, Campinas, SP, Brasil.
}

\section{Resumo}

competitividade da suinocultura brasileira no mercado mundial depende da sua adaptação às novas tendências e tecnologias. A questão do bem-estar animal, que é uma exigência dos mercados internacionais, tem gerado discussões e questionamentos sobre o sistema suinícola brasileiro atual. O sistema intensivo de criação apresenta diversos fatores geradores de estresse que comprometem o bem-estar dos suínos. Assim, o enriquecimento ambiental entra como uma ferramenta simples e efetiva que pode garantir o bem-estar dos animais em cativeiro. Enriquecer o ambiente consiste em fornecer artifícios aos animais que os estimulem a expressar o comportamento natural inerente à espécie, melhorando a sua saúde física e psicológica. O sistema de criação pode ser enriquecido de diversas maneiras, as quais instigam o interesse do animal de forma positiva, tais como disponibilização de brinquedos e substratos. Neste contexto, o objetivo deste artigo é revisar a relação do enriquecimento ambiental como o bem-estar de suínos, bem como as medidas que podem ser utilizadas para enriquecer o ambiente de criação.

Palavras Chave: brinquedos, comportamento animal, estresse, suinocultura

\begin{abstract}
The competitiveness of Brazilian pig production in the world market depends on their adaptation to global trends and technologies. Animal welfare as a requirement of international markets has generated discussion and questions about the current Brazilian pig farming. The intensive pig farming raises the most serious welfare concerns once it is surrounded by stress factors. Thus, environmental enrichment plays an important role as a simple and effective tool that can ensure the welfare of captive animal. Enrich an environment enhance the physical and psychological health by providing environmental stimuli necessary for encouraging the expression of natural inherent behaviors. Several stimuli which incite an animal's interest in a positive way can be used to enrich the housing systems, such as offering toys and substrate. This paper reviews the literature concerning environmental enrichment for pigs' welfare and some methods to enrich the pig housing system.
\end{abstract}

Keywords: animal behavior, toys, stress, swine production 


\section{INTRODUÇÃO}

A suinocultura brasileira tem se mostrado bastante competitiva no comércio mundial. A adaptação do país às novas tendências de mercado garante a sobrevivência deste na era globalizada.

A intensificação da produção de suínos em sistemas tradicionais de confinamento gera a polêmica do bem-estar animal ou da falta dele. No entanto, no Brasil, este tema tem pouca aplicação efetiva, uma vez que não há imposição do consumidor brasileiro. Por enquanto, a única situação de injunção do bem-estar animal na suinocultura brasileira seriam as exigências do mercado externo.

A definição de bem-estar é complexa e envolve vários fatores. Basicamente, é possível obter bem-estar positivo ao oferecer aos animais um ambiente de criação adequado, no qual ele possa expressar seus comportamentos naturais, ter longevidade e saúde. Como consequência, os animais poderão expressar seu potencial produtivo máximo.

Neste sentido, há dois tipos de medidas que podem ser utilizadas para melhorar as condições de bem-estar no sistema produtivo de suínos: as que interferem claramente no bem-estar (como exemplo a eliminação de procedimentos cruentos na criação) e as que colaboram com a amenização do estresse (tais como o enriquecimento ambiental).

Enriquecer um ambiente é fornecer aos animais artefatos permitindo que estes diversifiquem seus comportamentos. Ou ainda, um conceito mais amplo, segundo Dalla Costa et al. (2005), enriquecer um ambiente consiste em introduzir melhorias no próprio sistema confinado. O estudo do enriquecimento ambiental tem por objetivo proporcionar um ambiente adequado para criação, atendendo às necessidades comportamentais dos animais. Um animal com mais opções comportamentais terá mais chances de lidar com eventos estressantes ao seu redor (Pinheiro, 2009).

Dessa forma, proporcionar um ambiente no qual os suínos tenham liberdade de expressar o comportamento natural da espécie garante o bem-estar destes animais, por atender uma das cinco liberdades do bem-estar animal estabelecidos pela Farm Animal Welfare Council (1992).

Van de Weerd e Day (2009) enfatizam que proporcionar um ambiente com enriquecimento ambiental adequado para os suínos de todas as idades tem sido obrigatório entre os países da União Europeia (Diretrizes 2001/88/EC e 2001/93/EC). Nas diretrizes estão citados alguns tipos de materiais que podem ser usados para enriquecimento ambiental das baias, tais como palha, madeira, feno, serragem, turfa, composto de cogumelo e outros que não afetam a saúdes dos animais.

Considerando que o mercado europeu é um grande consumidor da carne suína brasileira, é importante que medidas de bem-estar, como enriquecimento ambiental sejam implantadas no sistema de criação.

O objetivo deste artigo é revisar as medidas que podem ser utilizadas para enriquecer o ambiente de criação bem com a relação do enriquecimento ambiental com bem-estar animal.

\section{BEM-ESTAR ANIMAL}

Atualmente, o bem-estar animal é um dos assuntos mais polêmicos, embora não seja um conceito novo. É de crescente importância no cenário suinícola mundial, principalmente por ser um requisito demandado pelos mercados internacionais consumidores da carne suína brasileira.

$\mathrm{Na}$ criação intensiva de suínos, os animais passam toda sua vida produtiva em instalações fechadas e com espaços individuais restritos, gerando situações que comprometem o bem estar.

Conceituar cientificamente bem-estar animal não é simples. Vários conceitos vêm sendo desenvolvidos, utilizados e aprimorados ao longo do tempo por diversos autores (BROOM, 1991; DUNCAN, 2005; YEATES e MAIN, 2008; SARUBBI, 2011; OHL E VAN DER STAAY, 2012). De modo geral, nas definições, bem-estar positivo indica ausência de sofrimento ou estresse e são baseadas nos princípios de bem-estar embasados nas cinco liberdades dos animais definidos pelo FAWC (Farm Animal WelfareCouncil, 1992): livres de fome e sede; livre de desconforto; livre de dor, sofrimento e doença; livre de medo e angústia; e livre para expressar seu comportamento natural.

Assim como o conceito, a avaliação do bem-estar na produção de suínos é complexa e envolve vários fatores, desde instalações, ambiente até o manejo (BAPTISTA et al., 2011). De acordo com Candiani et al. (2008), o bem-estar pode ser avaliado através de aspectos comportamentais, fisiológicos, sanitários e produtivos. Segundo Sarubbi (2011) outro conceito para quantificar o bem-estar é utilizando os princípios das "cinco liberdades".

Independente do conceito de bem-estar, é preciso inserir no sistema de criação medidas que visem melhorar o bem-estar dos animais, atendendo pelo menos o conceito das "cinco liberdades". 


\section{COMPORTAMENTO DE SUÍNOS}

O comportamento animal está intimamente interligado ao ambiente no qual os animais são criados, sendo fundamental nas adaptações das funções biológicas e, ainda, representa a parte do organismo que interage com o meio (SNOWDON, 1999).

Os suínos são animais onívoros, ou seja, sua fonte natural de comida é encontrada em uma área ampla. Assim, sob condições naturais eles passam grande parte do seu tempo explorando o ambiente à procura de alimento (STUDNITZ et a1., 2007). O comportamento exploratório é natural da espécie e importante para sua sobrevivência na natureza. Pinheiro (2009) caracteriza o comportamento exploratório como qualquer postura silenciosa e ações que indicam curiosidade, as quais incluem olhar, cheirar, lamber, fuçar ou mastigar um objeto. Grandin e Jonhson (2009) citam que em ambiente semi-natural, os suínos passam $52 \%$ do dia fuçando e pastando e $23 \%$ do tempo investigando o ambiente.

O suíno doméstico, embora seja alimentado pelos humanos, mesmo com toda evolução, ainda sente a necessidade de manifestar este tipo de comportamento. De acordo com Sarubbi (2011) quando os suínos não conseguem exercer o comportamento natural inerente da espécie, eles passam por um processo de frustação e direcionam o comportamento investigativo a outros animais ou equipamentos da baia.

Hötzel et al. (2010) mencionam um definição interessante de comportamento natural proposta por outros autores: "comportamento natural é aquele que o animal tende a realizar sob condições naturais, porque é prazeroso e promove bom funcionamento biológico".

No entanto, o sistema de criação comercial utilizado nos dias de hoje altera drasticamente estes comportamentos inerentes à espécie por propiciar um ambiente com situações diversas de estresse, tais como a separação da mãe após o desmame, o convívio social, a alta densidade, fome e baixa qualidade do ar nas instalações. Pandorfi (2005) relata que os suínos criados em condições artificiais e com restrições, sem contato com a natureza, apresentam com mais frequência comportamentos de estresse.

Os comportamentos de estresse, os quais comprometem o bem-estar, podem ser caracterizados como agonísticos e de frustração. Comportamentos como excessivo ato de fuçar, mastigação no vácuo, mamar e/ou morder a cauda ou orelha dos companheiros de baias, vocalização excessiva, pressionar o bebedouro sem beber água, podem caracterizar frustação. Campos et al. (2010) descrevem comportamento agonísticos como os animais brigando, mordendo ou arranhando um ao outro. A frustração dos animais leva a estereotipias (comportamentos repetitivos e sem função).

Sarubbi (2011) relata dois tipos de manifestações dos comportamentos estereotipados, as esporádicas ou contínuas. A primeira não é preocupante e ocorre em situações naturais, no entanto as continuas são indicadores de bem-estar pobre.

Os problemas comportamentais podem ser indicativos de deficiências ambientais e de manejo e, portanto, indicativos de alterações no bem-estar (SARUBBI, 2009). O autor indica que uma forma de estabelecer o manejo adequado à espécie é aumentando o conhecimento sobre seu comportamento social.

$\mathrm{Na}$ literatura científica, é possível verificar que em suínos sob condições de estresse térmico (QUINIOU et al., 1999; KIEFER et al., 2009), mistura de lotes ou super lotação de baias (SPOOLDER et al., 1999) ou quando alojados em ambientes áridos, monótonos e com falta de substratos (LOPES, 2004) podem ocorrer modificações no comportamento natural.

É necessário o conhecimento do comportamento dos animais para que os sistemas de criação animal sejam aprimorados, resultando em melhoria do bem-estar e do benefício econômico aos produtores (SOMMAVILLA, 2008).

\section{CONSIDERAÇÕES SOBRE ENRIQUECI- MENTO AMBIENTAL}

O bem-estar de animais de produção não é somente dependente da ausência de dor ou estresse, mas também considera pequenas modificações realizadas no ambiente de criação que garantem as necessidades comportamentais dos animais, que é uma das expressões das "Cinco Liberdades" (FAWC, 1992).

Entre as diversas vertentes para melhorar o bem-estar de suínos, uma importante é a chamada "enriquecimento ambiental", a qual consiste em introduzir melhorias no sistema de produção, com o objetivo de tornar o ambiente mais adequado às necessidades comportamentais dos animais (MACHADO FILHO e HÖTZEL, 2000).

Segundo Van de Weerd e Day (2009), o enriquecimento ambiental melhora o bem-estar dos animais por permitir que estes expressem seus comportamentos naturais específicos da espécie.

Campos et al. (2010) relatam que o enri- 
quecimento ambiental é um princípio do manejo animal que visa estender a qualidade de vida dos animais confinados, pelo fornecimento de estímulos ambientais que favorecem o bem-estar psíquico e fisiológico, por estimular suas necessidades etológicas.

Suínos que são alojados em ambientes áridos, monótonos, com falta de substratos, podem refletir frustração em comportamentos anômalos (LOPES, 2004).

Alguns autores (VAN DE WEERD et al., 2003; DAY et al., 2008; JENSEN et al., 2008; SARUBBI, 2011) citam que medidas de enriquecimento ambiental diminuem comportamentos indesejáveis, como agressividade e aumentam comportamentos naturais específicos da espécie suína, como o investigativo. Além disso, pode-se observar uma melhora na produtividade e sanidade e na qualidade do produto final, a carne suína (SARUBBI, 2011).

Estudos realizados com ratos em laboratório (FALKENBERG et al., 1992; ICKES et al., 2000) demonstram que o enriquecimento ambiental aumenta os níveis de neurotrofinas cerebrais, as quais estão associadas com a melhoria da aprendizagem espacial e aumento do comportamento exploratório.

Douglas et al (2012) estudaram o efeito do enriquecimento ambiental através da tendência cognitiva de suínos. Os autores verificaram que em ambientes enriquecidos os animais apresentavam estado emocional positivo, e consequentemente melhor bem-estar. E ainda, quando os suínos passavam algum tempo neste tipo de ambiente, reagiam negativamente quando alojados novamente em baias estéreis. Jansen et al. (2009) também encontraram efeito positivo do ambiente enriquecido sobre a aprendizagem e memória de suínos e sobre a manifestação do comportamento investigativo.

Neste contexto, entender o comportamento da espécie é fundamental para concepção, implantação e implementação do enriquecimento ambiental no processo de criação como medida de bem-estar. Por exemplo, considerando que os suínos perdem a atração por um objeto em um curto prazo de tempo, no planejamento do enriquecimento ambiental deve-se levar em consideração o tipo de objeto e a frequência de fornecimento e revezamento destes. Desconsiderar este comportamento pode afetar a finalidade de enriquecer o ambiente no sistema produtivo.

Resultados obtidos em um estudo realizado por Guy et al. (2013) comprovaram que os suínos se acostumam muito fácil a um determinado objeto. Da mesma forma, Zwicker et al. (2013), avaliando oito tipos de dispositivos de enriquecimento, enfatizaram a importância do valor em longo prazo dos materiais utilizados para eliciar o comportamento exploratório dos suínos em terminação.

Gifford et al. (2007) cita que uma maneira de renovar a novidade do objeto de enriquecimento é substituir o antigo por um novo objeto familiar.

O sistema de criação de suínos pode ser enriquecido de diversas formas. A disponibilização de substratos para cama (ex. palha), seguindo por objetos suspensos (ex. pneus e correntes), tem sido mais utilizado e tem por objetivo estimular o suíno a expressar seu comportamento natural de investigação e episódios de brincadeira, evitando assim frustação e comportamentos estereotipados. Por outro lado, existem outras formas de enriquecimento ambiental menos utilizadas na prática e também pouco estudadas cientificamente, mas que tendem a apresentar efeito positivo com relação ao bem-estar dos animais. Entre estas maneiras podemos citar a iluminação com diferentes cores de lâmpada e utilização de sons, aromas e ionizadores nas baias.

Bloomsmith et al. (1991) categoriza os tipos de enriquecimento em cinco classes: (1) Enriquecimento Social - envolve contato direto e indireto (sentidos sensoriais) com humanos ou outros indivíduos da mesma espécie; (2) Enriquecimento Ocupacional - engloba enriquecimento psicológico, como dispositivos que fornecem controle dos animais e enriquecimento que estimula o exercício; (3) Enriquecimento Físico - alterações no tamanho e complexidade das instalações, tais como oferecer objetos, substratos ou mudanças estruturais permanentes; (4) Enriquecimento Sensorial - utiliza dos sentidos sensoriais, exemplo, visual (televisão) e auditivo (musica e vocalização); (5) Enriquecimento Nutricional - disponibilizar variedades ou novos tipos de alimentos, assim como, mudança no método de fornecimento.

A escolha do tipo de enriquecimento a ser implantado geralmente é arbitrária, muitas vezes os critérios utilizados são relacionados a fatores econômicos e sanitários, sem considerar a necessidade do animal (VAN DE WEERD et al., 2003). É importante sopesar a escolha animal evitando que este perca o interesse rapidamente pelo objeto, o que pode limitar a sua funcionalidade como objeto de enriquecimento. Assim, os testes de preferência podem ser utilizados na avaliação de quais tipos de enriquecimento ou especificidade dos objetos usar a fim de obter o comportamento desejado e, também, para estabelecer alguns princípios sobre as características típicas da espécie frente aos diferentes tipos (BEATTIE et al., 1998; JENSEN et al., 
2008; BENCH et al., 2013; DEBOER et al., 2013)

Day et al. (2002) enfatizam que a eficácia e o sucesso de qualquer tipo de enriquecimento ambiental depende da sua capacidade de motivar um determinado comportamento típico da espécie.

Em revisão sobre enriquecimento ambiental para suínos em fase de terminação, Van de Weerd e Day (2009) sugeriram quatro características importantes do objeto de enriquecimento: (1) estimular o comportamento específico da espécie, (2) garantir a saúde física do animal; (3) ser de fácil implantação; (4) prover ganho econômico para sistema de produção.

Em alguns estudos é possível observar que materiais destrutíveis, variáveis, complexos, manipuláveis e com partes comestíveis, induzem a comportamentos típicos da espécie suína sendo que as duas primeiras características ainda estimulam a curiosidade dos animais e contribuem para prolongar o efeito de novidade (DAY et al., 2002; VAN DE WEERD et al., 2003; STUDNITZ et al., 2007; TRICKETT et al., 2009).

Outro ponto importante na escolha do dispositivo de enriquecimento é a idade dos animais. Docking et al. (2008) ressaltam que a idade dos suínos deve ser levada em consideração na hora da escolha do objeto de enriquecimento, uma vez que, a interação dos animais com este pode variar conforme a idade. Beattie et al. (2000) comentam que o enriquecimento ambiental é, geralmente, implementado após o desmame. No entanto, fatores que afetam a produtividade e a qualidade da carne nos suínos, como estresse, podem aparecer antes desta fase.

As pesquisas que visam avaliar o enriquecimento ambiental no sistema produtivo, geralmente, utilizam o comportamento dos animais como parâmetro (DAY et al., 2002; VAN DE WEERD et al., 2003; ZWICKER et al., 2013). No entanto, o efeito desta medida sobre o bem-estar de suínos pode ser avaliado usando diversas variáveis, tais como fisiologia e neurologia, parâmetros produtivos, qualidade da carne e sanidade (BEATTIE et al., 2000; DAY et al., 2002; SCOTT et al., 2006; 2007; CAMPOS et al., 2010).

\section{SUBSTRATOS PARA CAMA}

De acordo com pesquisas científicas (BEATTIE et al., 1998; MOINARD et al., 2003; VAN DE WEERD et al., 2006; ZWICKER et al., 2013), a forma de enriquecimento preferida pelos suínos são os substratos que podem ser utilizados como cama, principalmente a palha, por induzirem o comportamento investigativo, típico da espécie. Além disso, estes materiais podem ser trocados diariamente, mantendo seu valor de novidade/ atração.

Sarubbi (2011) relata que os substratos que formam cama para os animais são interessantes por estimularem o hábito de fuçar, investigar, chafurdar, além de reduzirem comportamentos indesejáveis, lesões originadas por luta e problemas locomotores.

Os tipos de substratos utilizados podem ser feno, maravalha, turfa, casca de arroz, palha e outros. Beattie et al. (1998) afirmam que materiais semelhantes a terra, como a turfa, podem ser objetos de enriquecimento mais efetivos. Resultados obtidos por Jensen et al. (2008) comprovam este relato.

Avaliando a preferência dos suínos por diferentes tipos e formas de materiais categorizados em seis grupos, Jensen et al. (2008) verificaram que entre as categorias estudadas, os animais somente expressaram preferência óbvia na categoria materiais tipo terra (compostagem $\mathrm{x}$ turfa $\mathrm{x}$ maravalha), preferindo compostagem e turfa à maravalha.

Beattie et al. (2000), enriquecendo baias de suínos do nascimento ao abate com turfa e palha, observaram que o enriquecimento reduziu o tempo em ócio e comportamentos agressivos. Por outro lado, o tempo gasto em comportamentos tipo exploratório foi maior, assim como, foi verificado maior taxa de crescimento, melhor conversão alimentar e qualidade da carcaça.

Van de Weerd et al. (2003) utilizaram um conjunto de 74 tipos de objetos e substratos diferentes em baias para suínos desmamados e em crescimento (exemplo: bolas, brinquedos de cachorro, correntes, espelhos e etc.) e observaram que o dispositivo mais popular foi a palha de lavanda. Em outro estudo, Van de Weerd et al. (2006) disponibilizando brinquedos e substratos, verificaram que a cama de palha foi definitivamente mais eficiente para distrair os suínos, e ainda, preveniu o comportamento de morder a cauda.

Chaloupková et al. (2007) aludiram que o enriquecimento ambiental com palha e maior espaço antes do desmame de leitões aumentou episódios de brincadeiras nesta fase e reduziu comportamentos agressivos de competição por alimento em fases posteriores, mas não afetou ocorrências de lutas durante o agrupamento dos animais após o desmame.

Averós et al. (2010) relataram menor incidência de comportamentos indesejáveis em baias com a disponibilidade de substrato para cama, além da diminuição do tempo em ócio e um menor nível de agressão entre os suínos. 
Durante um teste de motivação realizado com matrizes suínas, Elmore et al. (2012) disponibilizaram aos animais baias enriquecidas com brinquedos (borracha, tapete e cordas de algodão) e substratos (palha, compostagem e, verificaram menor agressividade dos animais devido ao enriquecimento e que entre os dispositivos oferecidos, as matrizes preferiram o composto e palha.

Em uma revisão sobre instalações de porcas gestantes alojadas em grupo do ponto de vista de bem-estar animal e produtividade, Bench et al. (2013) destacaram a importância do uso de substratos de cama como enriquecimento nesta fase de produção. Os autores citaram outros estudos que comprovaram que o uso de cama, principalmente de palha, no sistema produtivo melhora o conforto térmico e físico das matrizes suínas, diminuindo comportamentos de agressividade comuns em instalações em grupo. No entanto, desvantagens deste enriquecimento também foram destacadas, como aumento de custo e manejo, problemas sanitários e incompatibilidade com sistemas de drenagem de dejetos.

O enriquecimento ambiental usando substratos para cama tem sido a forma mais estudada pelos pesquisadores e também mais aplicada na prática. No entanto, a quantidade ideal de substrato a ser disponibilizada ainda não foi estabelecida satisfatoriamente pela ciência e pela prática. A maioria das leis e normas de boas práticas de criação só determina a utilização deste enriquecimento, sem determinar critérios para fornecimento.

Esta forma de enriquecimento ambiental, se comparada às outras formas supracitadas, necessita de um valor econômico maior para sua instalação. Para isso, a frequência e a quantidade a ser administrada devem ser mais bem estudadas por meio de análises de viabilidade. Devem ainda ser pesquisadas as relações entre o uso dos substratos e as condições climáticas locais (SARUBBI, 2011).

\section{BRINQUEDOS}

Embora os estudos tenham mostrado que os suínos preferem os substratos aos brinquedos (VAN DE WEERD et al., 2003; SCOTT et al., 2007; ELMORE et al., 2012), o uso de brinquedos como enriquecimento na suinocultura mostra-se bastante promissor, apresentando resultados significativos. Constituem-se artefatos simples que podem ser utilizados onerando pouco o custo de produção, por meio de ideias criativas, e ainda, reduzindo comportamentos que comprometem o bem-estar animal.
Os tipos de brinquedos mais comuns são pneus, correntes, garrafas pet, barras de madeira ou plástico, brinquedos de cães entre outros. Poucos testes têm sido realizados quanto à preferência dos suínos pelos tipos de brinquedos disponíveis para enriquecimento do ambiente de produção. A maioria das pesquisas comparam tipos de brinquedos com os substratos para cama (SCOTT et al., 2009; ELMORE et al., 2012)., tipo a palha, já que este último aparenta ser preferido pelos animais

Outros exemplos de objetos de enriquecimento podem ser obtidos em uma pesquisa de Van de Weerd et al. (2003), na qual os autores utilizaram 74 tipos de enriquecimento diferentes, considerando brinquedos e substratos. Como exemplo dos objetos utilizados podemos citar diversos tipos de bolas, espelho, baldes, tapete, peneira entre muitos outros.

Vale ressaltar que o valor de qualquer tipo de objeto de enriquecimento para o suíno irá depender de vários fatores, os quais incluem as características do material, grau de novidade, forma de fornecimento e outros. No entanto, poucos estudos têm avaliado se uso relativo ou absoluto do objeto é influenciado pelo contexto no qual ele é apresentado aos animais (GUY et al., 2013).

Em um estudo de preferência realizado por Jensen et al. (2008), três tipos de brinquedos suspensos (corda, bloco de madeira e cano de plástico) foram disponibilizados para os suínos. Os autores observaram que os animais não apresentaram interesse por nenhum dos objetos apresentados naquela condição. No entanto, eles referiram a outros estudos que forneceram os mesmos tipos de brinquedos, nos quais os animais preferiram a corda de sisal quando comparada a blocos de madeira e canos de plástico.

Nilsson (2012) concluiu que o uso de cordas como objeto de enriquecimento foi mais interessante para suínos do que bolas com comida de leitões.

As duas formas mais comuns de fornecimento dos tipos de brinquedos nas baias são suspensos ou no chão. Os suínos preferem materiais que estão suspensos a aqueles que são fornecidos no chão (BLACKSHAW et al., 1997; SCOTT et al., 2009; GUY et al., 2013). Blackshaw et al. (1997) sugeriram que o suíno perde o interesse primariamente pelos objetos sobre o chão por estes ficarem sujos mais rapidamente, perdendo a valor de novidade.

Resultados obtidos por Trickett et al. (2009) indicam que os grupos de suínos interagiram tanto com o bloco de madeira como com a corda de sisal, e concluíram que ambos os objetos apresentam 
alto valor de enriquecimento. Entretanto, quando o acesso a estes brinquedos foi contínuo, os animais interagiram mais com a corda e apresentaram mais atividades de exploração, por ser um objeto que atende mais as características desejáveis, tais como mastigável e destrutível. Além disso, a corda estava suspensa na baia, o que pode ter contribuído para a maior interação.

Ao combinarem quatro diferentes materiais de enriquecimento para suínos na fase de crescimento: objetos suspensos (corda de sisal e corrente de metal) e dois substratos (serragem e maravalha), Guy et al. (2013) verificaram que houve maior interação dos animais com a corda, seguido da corrente, serragem e por fim a marava1ha. E ainda, independente do material, o tempo no qual os animais gastavam interagindo com os objetos foi diminuindo ao longo do experimento, assim como, o nível de interação com os artefatos quando os animais eram apresentados a uma segunda combinação.

Em um teste de preferência individual conduzido com leitões, De Boer et al. (2013) enriqueceram as baias com tapete, companheiro da mesma espécie e espelho. De modo geral, os suínos preferiram passar maior parte do tempo no compartimento com o companheiro, e ainda, apresentaram maior frequência de comportamento investigativo. No entanto, na presença humana os animais preferiram o espelho igualmente ao companheiro. Os autores sugerem que o espelho pode ser utilizado pelo animal como apoio social durante períodos de ameaças.

Schaefer et al. (1990) utilizaram pneus suspensos como enriquecimento para suínos pós-desmame e verificaram que o enriquecimento reduziu agressão entre os animais e melhorou a taxa de crescimento. Resultados semelhantes foram obtidos por Pinheiro (2009). O autor também avaliou móbiles de pneu para leitões na fase pós desmame e concluiu que o enriquecimento ambiental diminui a frequência de comportamentos estereotipados e que a lavagem diária do pneu contribuiu para manter o interesse dos animais pelo objeto.

O uso de garrafa pet também foi estudado como brinquedo na suinocultura. Além de ser um artefato de baixo custo, usá-la como enriquecimento ambiental é uma forma de reutilizar este tipo de material, contribuindo com as questões ambientais.

Malheiros et al. (2010) utilizaram brinquedos suspensos confeccionados com garrafa pet na fase de desmame e observaram que no ambiente enriquecido os leitões reduziram o vício de morder, aumentaram os episódios de brincadeira e tiveram maior ganho de peso.

Em outro estudo realizado por Campos et al. (2010), o uso de garrafa pet como artefato para enriquecimento do ambiente na fase de creche, inicialmente, foi muito atraente para os animais que apresentaram evidência comportamental de melhor bem-estar, mas logo se acostumaram com o brinquedo e brincar com os outros leitões foi mais interessante.

Resultados preliminares obtidos por Zwirtes (2013) indicam que o uso de garrafas pet como enriquecimento ambiental de baias de leitões diminuiu a incidência de comportamentos agressivos. Os animais interagiram mais quando o brinquedo estava solto no chão do que suspenso, mas tiveram menor ganho de peso e maior frequência de comportamentos agonísticos.

Riesenberg et al. (2011) ao enriquecer baias de leitões alternando entre garrafas pet, pneus, correntes e bola, verificaram que os animais interagiram com todos os objetos, mas que preferiram a bola e a corrente. Embora não tenham encontrado melhora no desempenho produtivo, observaram menor estresse dos animais e diminuição do tempo de inatividade.

\section{MUSICOTERAPIA E AROMATERAPIA}

A utilização de estímulos sensoriais, como sons e aroma, pode ser apresentada como uma frente do bem-estar animal que colaboram com a amenização do estresse. Assim, a musicoterapia e a aromaterapia são formas de enriquecer um ambiente tornando-o menos monótono e contribuindo para o bem-estar positivo dos animais.

Estes artifícios como enriquecimento ambiental têm sido pouco estudados para a espécie suína, mas há resultados científicos promissores e benéficos ao bem-estar do ser humano e dos animais de estimação (ELLIS e WELLS et al., 2010; REINECKER; FAYAZI et al., 2011; MOREIRA et al., 2012).

A música é uma mistura complexa de notas, tons, amplitudes, ou seja, é um som contínuo e rítmico e por isso apresenta poder relaxante sobre o ser humano e os animais. Assim, a musicoterapia consiste em utilizar a música e seus elementos para reabilitação física, mental e social de indivíduos ou grupos. Segundo Moreira et al. (2012) a música transmite informações para o cérebro para serem processadas e esta ativação de certas regiões cerebrais são benéficas para estimular mudanças nas áreas cognitivas e motoras. 
Sarubbi (2011) relata que os sons são capazes de influenciar o comportamento dos animais e podem ser utilizados como reforço positivo e reforço negativo, o que está relacionado à capacidade cognitiva dos animais.

Estudos realizados com cães mostram que o estímulo musical acalmou os animais, reduzindo os latidos e sintomas de ansiedade de separação (TAKABATAKE et al., 2007; REINECKER et al., 2010). Para animais de produção, Albright e Arave (1997) verificaram que música clássica aumenta a produção leiteira. Jones et al. (2002) relatam que agricultores que fizeram o uso de rádio no sistema produtivo observaram redução de comportamentos agressivos, melhora da saúde e produtividade de aves.

Expondo suínos na fase de terminação a dois ritmos musicais (clássico e rock and roll), Ekachat e Vajrabukka (1994) observaram maior ingestão de matéria seca quando os animais foram submetidos ao rock and roll, porém o ganho de peso foi menor quando comparado aos animais tratados sem música.

Resultados obtidos por Jonge et al. (2008) apontam que leitões expostos à música antes do desmame, aumentaram os episódios de brincadeira na fase de creche e diminuiu comportamentos agressivos, constituindo um indicador positivo de bem-estar.

Resultados preliminares de pesquisadores brasileiros (MASSARI et al., 2010) apontam efeito da música clássica (Valsa das Flores de Peter Tchaikovsky) sobre o comportamento de leitões em fase de creche. Os animais que ouvem música durante a maternidade e a creche tem menor frequência de comportamento agonísticos, mas que durante o momento que a música tocava houve aumento dos episódios de luta.

Embora haja pouco fundamento científico com relação à musicoterapia, na prática, este método tem sido utilizado. Os produtores de suínos relatam que com a utilização de música os animais permanecem mais calmos, facilitando o manejo e diminuindo perdas de produtividade.

O suíno tem o olfato bastante desenvolvido e sensível, sendo o sentido olfativo vital para sua sobrevivência por estar relacionado a comportamentos básicos como comunicação, alimentação e reprodução. Assim, explorar esta característica pode ser útil ao usar o enriquecimento ambiental através de aromas estimulantes.

Aromaterapia visa melhorar a saúde física, emocional e espiritual dos seres vivos através do uso de óleos essenciais e outros compostos aromáticos extraídos de plantas. Estudos realizados mostram que extrato de lavanda e camomila diminuem a ansiedade, o estresse e a dor em humanos (FAYAZI et al., 2011; HADI e HANID, 2011), diminuem a vocalização e aumentam o tempo de descanso em cães (GRAHAM et al., 2005) e diminuem a frequência cardíaca em equinos (FERGUSON et al., 2012).

Jones et al. (2002) sugeriram a terapia olfativa para minimizar problemas comportamentais na criação de aves.

Com suínos, Wells (2009) relata os benefícios da utilização de feromônios, tais como redução de comportamento agressivo durante reagrupamento de animais usando cheiro de feromônios sexuais e diminuição de brigas e melhor ganho de peso no agrupamento de leitões pós desmame com uso de feromônios maternos.

Com suínos, Bradshaw et al. (1998) observaram que o uso de cama de palha aromatizadas com lavanda durante o transporte dos animais diminuiu a incidência e a severidade de mal estar, mas sem reduzir o estresse, medido pelas concentrações de cortisol salivar.

Krebs (2007) verificou que leitões desmamados expostos a aroma de banana e feromônio materno passaram mais tempo em repouso, e, ainda, os tratamentos exerceram um efeito positivo sobre o sistema imune. Por outro lado, não diminuiu a concentração de cortisol, hormônio indicador de estresse.

Van de Weerd et al. (2003) verificaram que a atração dos suínos por um determinado brinquedo foi aumentada quando estes foram aromatizados. Da mesma forma, Nowicki e Klocek (2012) avaliaram o efeito de brinquedos aromatizados como enriquecimento ambiental em baias de leitões desmamados e observaram que os animais foram atraídos pelo objeto, o que reduziu a frequência de agressividade entre os animais nos primeiros dias pós-desmame.

Ruchaber (2013) aspergiram óleo essencial de lavanda em sala de creche logo após o desmame e os resultados preliminares mostram que este enriquecimento contribuiu para diminuir os comportamentos indesejáveis como "brigas" e "vicio de morder".

\section{ILUMINAÇÃO}

Considerando que a reprodução dos suínos é influenciada pelo fotoperíodo, a importância da iluminação no sistema produtivo tem sido estudada como um fator ambiental de incremento da 
produtividade reprodutiva, como aparecimento da puberdade, taxa de concepção das fêmeas entre outros.

O número de horas de luz que os suínos necessitam por dia e a iluminância que atende as necessidades fisiológicas não são bem definidas cientificamente. Taylor et al. (2006) observaram que os animais preferem ambientes menos luminosos (2,4 contra 400 lux). Cocchi et al. (2009) indicam 80 lux para garantir o bem-estar dos animais, já a legislação europeia (CEE, 2008) recomenda o mínimo de 40 lux. A Humane Farm Animal Care (HFAC, 2008) indica iluminação mínima de 50 lux.

Poucos estudos focam na iluminação como enriquecimento ambiental, mas este artifício pode ter efeitos interessantes sobre o bem-estar dos animais.

Souza et al. (2011a) observaram preferência dos leitões na maternidade por escamoteadores iluminados com lâmpadas de cor azul quando comparado com lâmpada branca. Também observaram comportamentos de curiosidade em leitões de maternidade nos primeiros minutos quando os escamoteadores com lâmpadas azuis foram acessos. Em outro estudo, Souza et al. (2011b) avaliando o comportamento de leitões em fase de creche submetidos ao escamoteador enriquecido com lâmpada de cor azul, observaram que os animais ficaram mais agitados, ou seja, os animais visitaram mais vezes este escamoteador durante o período estudado, devido à preferência dos leitões pelo mesmo. Ficou suposto que o tipo de enriquecimento ambiental utilizado na maternidade pode atrair mais os leitões para este ambiente. Os autores ainda afirmam que o escamoteador com luz azul pode ter favorecido o bem-estar dos leitões por não ter emitido brilho excessivo a visão, não causando o ofuscamento de sua visão. Porém, os autores discutem que a preferência pode ter sido atribuída pela menor iluminância gerada no interior do abrigo pela lâmpada de cor azul, quando comparada à lâmpada de cor branca.

Taylor et al. (2006), estudando preferência de iluminância para suínos em crescimento, observaram que os animais preferem áreas menos iluminadas para descansar e, mais iluminadas para defecar. Desta forma, os estudos sobre a necessidade dos animais quanto à iluminação podem ser interessantes para melhorar a higiene das baias e melhorar o desempenho e bem-estar dos animais, havendo a possibilidade de criar zonas mais iluminadas para defecação e menos iluminadas para descanso.

No trabalho de Amaral (2012), com programa de iluminação para suínos em terminação, concluíram que o fornecimento de luz adicional no período do dia influenciou o comportamento de suínos que ficaram mais ativos em suas atividades.

\section{IONIZAÇÃO DO AR}

O uso de ionizadores nas instalações constitui um tipo promissor de enriquecimento ambiental que parece ter influência sobre a qualidade do ar do ambiente, melhorando assim a saúde e bem-estar do animal. A redução de partículas de poeira e gases prejudiciais do ar das instalações suinícolas é muito importante tanto para saúde do animal, como para o trabalhador.

A ionização é o resultado de um processo pelo qual uma molécula eletricamente neutra adquire uma carga elétrica positiva ou negativa. A literatura mostra que o uso de íons negativos produz efeitos benéficos para humanos, como relaxamento, redução do cansaço, estresse e ansiedade, enquanto a íons positivos causa efeitos nocivos, por exemplo irritabilidade e aumento do hormônio de estresse (OGUNGBE et al., 2011; PEREZ et al., 2013).

Para animais, há pouca literatura a respeito, principalmente relacionado ao efeito da ionização do ar sobre os animais. A maioria dos trabalhos realizados enfatiza o efeito da ionização na melhoria da qualidade do ar das instalações.

Mitchell et al (2004), aplicando íons negativos em instalações para matrizes de corte, observaram redução da quantidade de poeira, amônia e bactérias no ar. Tanaka e Zhang (1996) e Ritz et al. (2006) também conseguiram reduzir a concentração de poeiras em instalações para suínos e aves, respectivamente.

Avaliando o efeito da ionização na redução de partículas e gases emitidos em galpões de frangos, Cambra-Lopez et al (2009) verificaram que adição de íons negativos reduziu o tamanho das partículas, mas não teve efeito na redução dos gases e odores, assim como no desempenho dos animais.

Geng et al. (2011) estudaram o efeito da ionização sobre a qualidade do ar em granjas de suínos e verificaram a ionização reduziu a concentração de endotoxinas nas instalações em $38,5 \%$, além de reduzir, as concentrações de gás carbônico e micro-organismos nocivos à saúde. Resultados semelhantes foram obtidos por Sarubbi et al. (2011). Os autores utilizaram ionização negativa em instalações de creche e encontraram diminuição nas concentrações de amônia e gás carbônico, porém não houve efeito sobre o com- 
portamento dos animais.

\section{OUTROS TIPOS DE ENRIQUECIMENTO AMBIENTAL}

Além das formas supracitadas, há outros artifícios menos estudados que podem ser utilizados para enriquecer o ambiente e melhorar o bem-estar de suínos.

Sarubbi (2011) comenta do enriquecimento estrutural nas baias, tais como baia com dois níveis de piso, baia enriquecida com mezanino e uso de lâminas d'água. Dalla Costa et al. (2005) também consideram como medidas de enriquecimento ambiental: utilização de baias coletivas para porcas em gestação, melhora nas celas de parição, manejo diários com os animais (relacionamento tratador e animal). Outros estudos mencionam como enriquecimento ambiental o aumento de espaço das baias e áreas livres na instalação (CHALOUPKOVÁ et al., 2007; TALLET et al., 2013).

Considerando a relação social da espécie suína, garantir a companhia de leitões irmãos e fornecer úbere artificial quente com mamilos flexíveis pode diminuir o estresse em leitões desmamados (COLSON et al., 2006; BENCH e GONYOU, 2007). Além da companhia de animais da mesma espécie, o contato com manejador é muito importante. Eles devem desenvolver uma atitude positiva frente aos suínos, movendo os animais com calma, evitando movimentos bruscos, agachando-se para reduzir o tamanho do corpo aparente, e no manuseio pode se acariciar ou coçar o corpo dos animais (HEMSWORTH et al, 1996). Assim, os suínos vão se aproximar com confiança e interagir com os tratadores de forma positiva.

Barreiras visuais ajudam os animais a evitarem comportamentos agressivos quando alojados em grupo, por exemplo, instalação de divisórias entre diferentes áreas funcionais (WARAN e BROOM, 1993; ANDERSEN et al., 1999) ou uso de rampas (PEDERSEN, 1992). Em baias externas o uso de arbustos e árvores pode criar áreas visualmente agradáveis aos animais. Lewis et al. (2008) comentam que o uso de rampas ou baias com dois níveis de piso pode reduzir o comportamento de medo quando os animais forem manejados para o abate.

A utilização de piso resfriado para porcas na maternidade (WAGENBERG et al., 2006; FERNANDES et al., 2011; LIMA et al., 2011) e de piso aquecido para leitões lactantes (FERNANDES et al., 2011; SABINO et al., 2011 SARUBBI et al., 2010) também constitui um tipo de enriquecimento estrutural que proporciona conforto térmico para os animais, evitando comportamentos de estresse e consequente melhora no desempenho produtivo.

O uso de um tapete sintético para descanso de porcas em gestação foi avaliado por TUYTTENS te al. (2008). Os autores verificaram preferência dos animais pelo tapete se comparado com o piso de concreto, uma vez que este proporcionou melhor conforto térmico. Semelhantemente, em estudo mais recente, GU et al. (2010) evidenciara que o uso de tapetes de neoprene nas gaiolas de parto, debaixo da matriz e na área de amamentação dos leitões, propiciou melhora no conforto térmico, saúde e bem estar dos animais, assim como melhora no manejo de dejetos.

\section{CONSIDERAÇÕES FINAIS}

O uso do enriquecimento ambiental como medida para melhorar o bem-estar de suínos confinados é promissor e eficiente. Há métodos, como disponibilização de substratos para cama e brinquedos, que possuem respaldo científico, embora ainda haja muitos critérios a serem estudados e definidos. Outros métodos, tais como ionização do ar, musicoterapia e aromaterapia, que são menos pesquisados, prometem resultados satisfatórios.

A escolha do tipo de enriquecimento a ser utilizado em uma granja é subjetiva, sendo dependente da sua realidade. É importante considerar a viabilidade econômica e as condições de operacionalização do enriquecimento na unidade de produção, ressaltando que o enriquecimento ambiental pode ser uma ferramenta de baixo custo e de bons resultados, desde que se use a criatividade.

\section{REFERÊNCIAS BIBLIOGRÁFICAS}

\author{
ALBRIGHT, J. L.; ARAVE, C. W. The behaviour of \\ cattle.1997.320 pp.
}

AMARAL, P.I.S. Programa de iluminação para suínos em terminação 2012.34p. Dissertação (Mestrado em Zootecnia), Universidade Federal dos Vales do Jequitinhonha e Mucuri,Diamantina, 2012.

ANDERSEN, I.L.; BOE, K.E.; KRISTIANSEN, A.L. The influenceof different feeding arrangements and food type on competition at feeding in pregnant sows. Applied Animal Behaviour Science, Elsevier, Amsterdam, v. 65, n. 2, p. 91-104, Oct, 1999. 
AVERÓS, X. et al. A meta-analysis of the combined effect of housing and environmental enrichment characteristics on the behaviour and performance of pigs. Applied Animal Behaviour Science, Elsevier, Amsterdam, v.127, n. 3-4, p. 73-85, Nov., 2010.

BAPTISTA, R.I.A.A.; BERTANI, G.R.; BARBOSA, C.N. Indicadores do bem-estar em suínos. Ciência Rural, Santa Maria, v. 41, n. 10, p. 1823-1830, Oct., 2011.

BEATTIE, V.E., WALKER, N., SNEDDON, I.A., Preference testing of substrates by growing pigs. Animal Welfare, Hertfordshire, v.7, n.1, p.27-34, Feb., 1998.

BEATTIE, V. E.; O'CONNELL, N.E.; MOSS, B.W. Influence of environmental enrichment on the behavior, performance and meat quality of domestic pigs. Livestock Production Science, Elsevier, Amsterdam, v. 65, n. 1-2, p. 71-79, Jul., 2000.

BENCH, C.J.; GONYOU, H.W. Effect of environmental enrichment and breed lineon the incidence of belly nosing in pigletsweaned at 7 and 14 days-of-age. Applied Animal Behaviour Science, Elsevier, Amsterdam, v.105, n. 1-3, p. 26-41, Jun., 2007.

BENCH, C.J. et al. Group gestation sow housing with individual feeding - II: How space allowance group size and composition, and flooring affect sow welfare. Livestock Science, Elsevier, Amsterdam, v. 152, n. 2-3, p. 218-227, Apr., 2013.

BLACKSHAW, J.K. et al. The effect of a fixed or free toy on the growth rate and aggressive behaviour of weaned pigs and the influence of hierarchy on initial investigation of the toys. Applied Animal Behaviour Science, Elsevier, Amsterdam, v. 53, n. 3, p. 203-212, Jun., 1997.

BLOOMSMITH, M. A., BRENT, L.Y.; SCHAPIRO, S.J. Guidelines for developing and managing an environmental enrichment program for nonhuman primates. Laboratory Animal Science, Memphis, $\mathrm{n}$. 41, v. 4, p. 372-377, Aug., 1991.

BRADSHAW, R. H. et al. Effects of lavender Straw on Stress and Travel Sickness in Pigs. The Journal of Alternative and Complementary Medicine, New Rochelle, v. 4, n. 3, p. 271-275, Fall, 1998.
BROOM, D. Animal welfare: concepts and measurements. Journal of Animal Science, Champaign, v. 69, v. 10, p. 4167-4175, Oct., 1991.

CAMBRA-LOPEZ, M. et al. Ionization for reducing particulate matter emissions from poultry houses. Transactions of the ASABE, v. 52, p. 1757-1771, 2009.

CANDIANI, D. et al. A combination of behavioral and physiological indicators for assessing pig welfare on the farm. Journal of Applied Animal Welfare Science, Ann. Arbor., v.11, n.1, p.1-13, 2008.

CHALOUPKOVÁ, H. et al. The effect of pre-weaning housing on the play andagonistic behaviour of domestic pigs. Applied Animal Behaviour Science, Elsevier, Amsterdam, v.103, n. 1-2, p. 25-34, Mar., 2007.

CAMPOS, J.A.; TINÔCO, I.F.F., SILVA, F.F.; et al. Enriquecimento ambiental para leitões na fase de creches advindos de desmame aos 21 e 28 dias. Revista Brasileira de Ciências Agrárias, Recife, v. 5, n. 2, p. 272-278, abr-jun, 2010.

CEE. Comunidade Econômica Européia. Directiva 2008/120/CE. Dezembro de 2008. Relativa às normas mínimas de protecção de suínos. 2008. Disponível em: <http://eur-lex.europa.eu/LexUriServ/ LexUriServ.do?uri=OJ:L:2009:047:0005:0013:PT: PDF>. Acessoem: 15 mar. 2013.

COCCHI, M. et al. Do mood disorders play a role in pig welfare? Italian Journal of Animal Science, Pavia, v. 8, n. 4, p. 691-704, 2009.

COLSON, V. et al. Grouping piglets by sex at weaning reduces aggressive behaviour. Applied Animal Behaviour Science, Elsevier, Amsterdam, v. 97, n. 1-2, p. 152-171, May, 2006.

DALLA COSTA, O.A. et al. Aspectos econômicos e de bem-estar animal no manejo dos suínos da granja até o abate. In: SEMINÁRIO INTERNACIONAL DE AVES E SUÍNOS, 4, 2005, Florianópolis. Anais... Disponível em: <http://www.agencia.cnptia. embrapa.br/recursos/publicacao_c7t41d7n_pre_abateID-WyUdT5iwKc.pdf> Acesso em: 01 mai. 2013.

DAY, J.E.L. et al. The separate and interactive effects of handling and environmental enrichment on the behaviour and welfare of growing pigs. Applied Animal Behaviour Science, Elsevier, Amsterdam, v. 75, n. 3; p. 177-192, Jan., 2002. 
DAY, J.E.L. et al. The effect of varying lengths of straw bedding on the behaviour of growing pigs. Applied Animal Behaviour Science, Elsevier, Amsterdam, v. 109, n. 2-4, p. 249-260, Feb., 2008.

DEBOER, S.P. et al. Does the presence of a human affect the preference of enrichment items in young, isolated pigs? Applied Animal Behaviour Science, Elsevier, Amsterdam, v. 143, n. 2-4, p. 96-103, Jan., 2013.

DOCKING, C.M. et al. The influence of age on the use of potential enrichment objects and synchronisation of behaviour of pigs. Applied Animal Behaviour Science, Elsevier, Amsterdam, v. 110, n. 3-4, p. 244257, Apr., 2008.

DOUGLAS, C. et al. Environmental enrichment induces optimistic cognitive biases in pigs. Applied Animal Behaviour Science, Elsevier, Amsterdam, v.139, n.1-2, p.65-73, Jun., 2012.

ELMORE, M.R.P. et al. Differing results for motivation tests and measures of resource use: The value of environmental enrichment to gestating sows housed in stalls. Applied Animal Behaviour Science, Elsevier, Amsterdam, v. 141, n. 1-2, p. 9-19, Oct., 2012.

DUNCAN, I.J.H. Science-based assessment of animal welfare: farm animals. Rev. Sci. Tech. Off. Int. Epiz, v. 24, n. 2, p. 483-492, 2005. Disponível em: <http://www.labea.ufpr.br/publicacoes/pdf/ Tradu\%E7\%E3o\%20LABEA\%20WSPA\%20Duncan. pdf> Acesso em: 24 abr. 2010.

EKACHAT, K., VAJRABUKKA, C. Effect of music rhythm on growth performance of growing pigs.

Kasetsart Journal Natural Science, Bangkok, v. 28, n. 4, p. 640-643, oct-dec, 1994.

ELLIS, S. H.; WELLS, D. L. The influence of olfactory stimulation on the behaviour of cats housed in a rescue shelter. Applied Animal Behaviour Science, Elsevier, Amsterdam, v. 123, n. 1, p. 56-62, Feb., 2010.

FAYAZI, S.; BABASHAHI, M.; REZAEI, M. The effect of inhalation aromatherapy on anxiety level of the patients inpreoperative period. Iranian Journal of Nursing and Midwifery Research, v. 16, n. 4, p. 278283, Autumn, 2011.

FALKENBERG, T. A. K. et al. Increased expression ofbrain-derived neurotrophic factor mRNA in rat hippocampus isassociated with improved spatial memory and enriched environment. Neuroscience Letters, Elsevier, Amsterdam, v. 138, n. 1, p. 153156, Apr., 1992.

FAWC - FARM ANIMAL WELFARE COUNCIL. Updates the five freedoms. The Veterinary Record, London, v. 131, p. 357, 1992.

FERNANDES, H.C. et al. Efeito do aquecimento e resfriamento de pisos no desempenho de matrizes e leitões. Revista Ceres, Viçosa, v. 58, n. 6, p. 701-709, nov/dez, 2011.

GENG, Q; GILBERTSSON, M.; NILSSON, M. Effect of ionization on air quality in a pig house pilot study. In: INTERNATIONAL CONFERENCE ON BIOINFORMATICS AND BIOMEDICAL ENGINEERING (ICBBE), 5th, 2011. Proceedings... Disponível em: <http://ieeexplore.ieee.org/xpl/ articleDetails.jsp?reload=true $\&$ arnumber $=5781238>$. Acessoem: 02 nov. 2012.

GIFFORD, A.K.; CLOUTIER, S.; NEWBERRY, R.C. Objects as enrichment: Effects of object exposure time and delay interval on object recognition memory of the domestic pig. Applied Animal Behaviour Science, Elsevier, Amsterdam, v. 107, n. 3-4, p. 206-217, Nov., 2007.

GRAHAM, L.; WELLS, D.L.; HEPPER, P.G. The influence of olfactory stimulation on the behaviour of dogs housed in a rescue shelter. Applied Animal Behaviour Science, Elsevier, Amsterdam, v. 91, n. 1-2, p. 143-153, May, 2005.

GRANDIN, T.; JOHNSON, C. Bem-estar dos animais. São Paulo: Rocco, 2009. 336p.

GU, Z. et al. Effects of neoprene mat on diarrhea, mortality and foreleg abrasion ofpre-weaning piglets. Preventive Veterinary Medicine, Elsevier, Amsterdam, v. 95, n. 1-2, p. 16-22, Jun., 2010.

GUY, J.H.; MEADS, Z.A.; SHIEL, R.S. et al. The effect of combining different environmental enrichment materials on enrichment use by growing pigs. Applied Animal Behaviour Science,Elsevier, Amsterdam, v. 144, n.3, p. 102-107, Mar., 2013.

HFAC - HUMANE FARM ANIMAL CARE

-.Padrões dos Cuidados com Animais: Porcos. 2008. Disponível em: <http://www.certifiedhumane.org/ pdfs/Std08Porcos1R_po.pdf.> Acesso em: 15 mar. 2013. 
HEMSWORTH, P.H.; PRICE, E.O.; BORGWARDT, R. Behavioural responses ofdomestic pigs and cattle to humans and novel stimuli. Applied Animal Behaviour Science, Elsevier, Amsterdam, v. 50, n. 1 p. 43-56, oct., 1996.

HÖTZEL, M.J.; NOGUEIRA, S.S.C.; MACHADO FILHO, L.C.P. Bem-estar de animais de produção: das necessidades animais às possibilidades humanas. Revista de Etologia, São Paulo, v. 9, n. 2, p. 1-10, Dez., 2010.

ICKES, B.R. et al. Long-Term Environmental Enrichment Leads to Regional Increases in Neurotrophin Levels in Rat Brain. Experimental Neurology, Elsevier, Amsterdam, v. 164, n. 1, p. 45-52, 2000.

JANSEN, J. et al. Spatial learning in pigs: effects of environmental enrichment and individual characteristics on behaviour and performance. Animal Cognition, Heidelberg, v. 12, p. 303-315, 2009.

JENSEN, M.B. et al. Pigs' preferences for rooting materials measured in a three-choice maze-test. Applied Animal Behaviour Science, Elsevier, Amsterdam, v. 112, n. 3-4, p. 270-283, Aug., 2008.

JONES, B.R. Role of Comparative Psychology in the Development of Effective Environmental Enrichment Strategies to Improve Poultry Welfare. International Journal of Comparative Psychology, v. 15, n. 2, p. 77-106, 2002.

JONGE, H. F. et al. Music during play-time: Using context conditioning as a tool to improve welfare in piglets. Applied Animal Behaviour Science, Elsevier, Amsterdam,v. 15, n. 3-4, p. 138-148, Dec., 2008.

HADI, N.; HANID, A.A. Lavender Essence for Post-cesarean Pain. Pakistan Journal of Biological Sciences, v.14, n. 11, p. 664-667, Jun., 2011.

KIEFER, C. et al. Resposta de suínos em crescimento mantidosem diferentes temperaturas. Revista Archivos de Zootecnia, Córdoba, v.58, n. 221, p. 55-64, Mar., 2009.

KREBS, N. Odors and Pheromones: Influences of Olfaction on Behavior, Physiology, and Performance to reduce Stress in Pigs. 2007. 172p. Dissertation (Doctor of Philosophy in Animal Science) - Texas Tech University, Lubbock, 2007.

LEWIS, C.R.G; HULBERT, L.E.; McGLONE. J.J.Novelty causes elevated heart rate and immune changes in pigs exposed to handling, alleys, and ramps. Livestock Science, Elsevier, Amsterdam, v. 116, n. 1-3, p. 338-341, Jul., 2008.

LIMA, A.L. et al. Resfriamento do piso da maternidade para porcas em lactação no verão. Revista Brasileira de Zootecnia, Viçosa, v. 40, n. 4, p. 804811, Abr., 2011

LOPES, E.J.C. Análise do bem-estar e desempenho de suínos em sistema de cama sobreposta. 2004. 111p. Dissertação (Mestrado em Agroecossistemas) - Curso de Pós-graduação em Agrossistemas, Universidade Federal de Santa Catarina, SC.

MACHADO FILHO, L.C.P., HÖTELZ, M.J. Bem-estar dos suínos. In: SEMINÁRIO INTERNACIONAL DE SUINOCULTURA, 5, 2000, São Paulo. Anais... São Paulo, p. 1-13.

MALHEIROS, F.M. et. al. Efeitos da utilização de brinquedo suspenso confeccionado com garrafa pet no comportamento e desempenho zootécnico de suínos em fase de creche. In: PORK EXPO - FÓRUM INTERNACIONAL DE SUINOCULTURA, V., 2010., Curitiba. Anais... Campinas: Animal Word, 2010.

MASSARI, J.M. et. al. Efeito da música no bem-estar animal de suínos na fase de creche. In: PORK EXPO - FÓRUM INTERNACIONAL DE SUINOCULTURA, V, 2010., Curitiba. Anais...Campinas: Animal Word, 2010.

MITCHELL, B.W.; RICHARDSON, L.J.; WILSON, J.L.; HOFACRE, C.L. Application of an electrostatic space charge system for dust, ammonia, and pathogen reduction in a broiler breeder house. Applied Engineering in Agriculture, St. Joseph, v. 20, p. 87-93, 2004.

MOINARD, C. et al. A case control study of on-farm risk factors for tail biting in pigs. Applied Animal Behaviour Science, Elsevier, Amsterdam, v. 81, n. 4, p. 333-355, May, 2003.

MOREIRA, S.V. Neuromusicoterapia no Brasil: aspectos terapêuticos na reabilitação neurológica. Revista Brasileira de Musicoterapia, ano XIV, n. 12, p. 18-26, 2012.

NILSSON, E. The effects of an activity ball and ropes on pigs' behaviours. 16p. Thesis. Department of Physics, Chemistry and Biology, Linköpings Universitet, Linköpings, 2012. 
NOWICKI, J.; KLOCEK, C. The effect of aromatized environmental enrichment inpen on social relations and behavioural profile of newly mixed weaners. Annals of Animal Science, Krakow, v. 12, n. 3, p. 403-412, Jul., 2012.

OGUNGBE, A.S.; AKINTOYE, O.H.; IDOWU, B.A. Effects of gaseous ions on the environment and human performance. Trends in Applied Sciences Research, New York, v. 6, n. 2, p.130-133, 2011.

OHL, F.; VAN DER STAAY, F.J. Animal welfare: at the interface between science and society. The Veterinary Journal, Elsevier, Amsterdam, v. 192, n. 1, p. 13-19, Apr., 2012.

PANDORFI, H. Comportamento bioclimático de matrizes suínas em gestação e o uso de sistemas inteligentes na caracterização do ambiente produtivo: suinocultura de precisão. 2005. 119p. Tese (Doutorado em Agronomia) - Escola Superior de Agricultura "Luiz de Queiroz", Universidade de São Paulo, Piracicaba, 2009.

PEDERSEN, B.K. Comprehensive evaluation of well-being in pigs: Environmental enrichment and pen space allowance. 1992. 195p. Dissertation (Doctor of Philosophy in Animal Sciences) - Graduate College of University of Illinois at Urbana-Champaign, Urbana-Champaign, 1992.

PEREZ, V.; ALEXANDER, D.D.; BAILEY, W.H. Air ions and mood outcomes: a review and meta-analysis. BMC Psychiatry, London, v. 13, n. 29, [S.p]; Jan, 2013.

PINHEIRO, J.V. A pesquisa com bem estar animal tendo como alicerce o enriquecimento ambiental através da utilização de objeto suspenso no comportamento de leitões desmamados e seu efeito como novidade. 2009.65p. Dissertação (Mestrado em Nutrição e Produção Animal), Faculdade de Medicina Veterinária e Zootecnia, Universidade de São Paulo, São Paulo, 2009.

QUINIOU, N.; NOBLET, J. Influence of high ambient temperatureon performance of multiparous lactating sows. Journal of Animal Science, Champaign, v. 77, n. 8, p. 2124-2134, Aug., 1999.

REINECKER, D. Music for dogs. Disponível em: $<$ http://www.learningdog.com/musicfordogs.html $>$. Acesso em: 08 ago. de 2012.
RIESENBERG, A.A. et al. Enriquecimento ambiental na forma de brinquedos para leitões após o desmame. Disponível em: <http://inf.ifc-araquari. edu.br/mct/wp-content/uploads/2011/11/Enriquecimento-ambiental-na-forma-de-brinquedos-para-leit\%C3\%B5es-ap\%C3\%B3s-o-desmame.pdf> Acesso em: 30 abr. 2013.

RITZ, C.W. et al. Improving in-house air quality in broiler production facilities using an electrostatic space charge system. The Journal of Applied Poultry Research, Champaign, v. 15 , n. 2, p. 333-340, Summer, 2006.

RUCHABER, C.M. Efeito do uso da aromaterapia em leitões após o desmame. 2013. 40p. Monografia (Curso de Graduação em Zootecnia) - Universidade Federal de Santa Maria, Palmeira das Missões, 2013.

SABINO, L.A. et al. Comportamento suíno influenciado por dois modelos de maternidade. Revista Brasileira de Engenharia Agrícola e Ambiental, Campina Grande, v. 15, n. 12, p. 1321-1327, 2011.

SARUBBI, J. Bem-estar dos animais e uso racional de energia elétrica em sistemas de aquecimento para leitões desmamados. 2009.190p. Tese (Doutorado em Engenharia Agrícola), Faculdade de Engenharia Agrícola, Universidade Estadual de Campinas, Campinas, 2009.

SARUBBI, J. et al . Utilização de energia elétrica em diferentes sistemas de aquecimento para leitões desmamados. Engenharia Agrícola, Jaboticabal, v. 30, n. 6, Dec, 2010.

SARUBBI, J. Bem estar animal não se restringe às instalações e equipamentos: o uso de novas tecnologias. In: FÓRUM INTEGRAL DE SUINOCULTURA: TEORIA E PRÁTICA DO BEM ESTAR ANIMAL NA PRODUÇÃO DE SUÍNOS, 1, 2011, Curitiba. Anais... p. 36-50.

SARUBBI, J.; LORINI, D.; MOURA, D.J.; MAIA, A.P.A.; MEDEIROS, B.B.L. Ionização de instalações para suínos em fase de creche: efeitos na qualidade do ar. In: CONGRESSO ABARAVES, XV, 2011, Fortaleza. Anais... Fortaleza: ABRAVES, 2011.

SCOTT, K. et al. Influence of different types of environmental enrichment on the behaviour of finishing pigs in two different housing systems: 1 . Hanging toy versus rootable substrate. Applied Animal Behaviour Science, Elsevier, Amsterdam, v. 99, n. 3-4, p. 222229, Sept., 2006. 
SCOTT, K. et al. Influence of different types of environmental enrichment on the behaviour of finishing pigs in two different housing systems. 2. Ratio of pigs to enrichment. Applied Animal Behaviour Science, Elsevier, Amsterdam, v. 105, n. 1-3, p. 51-58, Jun., 2007.

SCOTT, K. et al. Influence of different types of environmental enrichment on the behaviour of finishing pigs in two different housing systems: 3 . Hanging toy versus rootable toy of the same material. Applied Animal Behaviour Science, Elsevier, Amsterdam, v. 116, n. 2-4, p. 186-190, Jan., 2009.

SCHAEFER, A.L. et al. The effect of environment enrichment on aggression in newly weaned pigs. Applied Animal Behaviour Science, Elsevier, Amsterdam, v. 27, n. 1-2, p. 41-52, Aug., 1990.

SOUZA, S. et al. Teste de preferência de leitões submetidos a escamoteadores enriquecidos com lâmpadas de cor azul. In: CONGRESSO ABRAVES, XV, 2011, Fortaleza. Anais... Fortaleza: ABRAVES, 2011a.

SOUZA, S. et al. Comportamento e preferência de leitões lactentes submetidos ao escamoteador enriquecido com lâmpadas de cor azul. In: CONGRESSO ABRAVES, XV, 2011, Fortaleza. Anais... Fortaleza: ABRAVES, 2011b.

SNOWDON, C. T. O significado da Pesquisa em Comportamento Animal. Estudo de Psicologia; São Paulo, v. 4, p.365-373, 1999.

SOMMAVILLA, R. Comportamento e bem-estar de animais zootécnicos. 2008. 69p. Relatório de Estágio Curricular Obrigatório- Medicina Veterinária. Universidade do Estado de Santa Catarina, Centro de Ciências Agroveterinárias, Lages.

SPOOLDER, H. A. M.; S. A. EDWARDS, S. CORNING. Effects of group size and feeder space allowance on welfare in finishing pigs. Journal of Animal Science, Champaign, v. 69, p. 481-489, 1999.

STUDNITZ, M., JENSEN, M.B., PEDERSEN, L.J. Why do pigs root and in what will they root? A review on the exploratory behaviour of pigs in relation to environmental enrichment. Applied Animal Behaviour Science, Elsevier, Amsterdam, v. 107, n. 3-4, p. 183-197, Nov., 2007.

TALLET, C. et al. Effects of neonatal castration on social behaviour, human-animal relationship and feeding activity in finishing pigs reared in a conventional or an enriched housing. Applied Animal Behaviour Science, Elsevier, Amsterdam, v. 145, n. 3, p. 70-83, May, 2013.

TANAKA, A.; ZHANG, Y. Dust Settling Efficiency and Electrostatic Effect of a Negative Ionization System. Journal of Agricultural Safety and Health, St. Joseph, v. 2, p. 39-47,1996.

TAKABATAKE, E.Y. A Musicoterapia para cães com depressão. 2007. 36p. Monografia (Graduação do Curso de Musicoterapia) - Faculdade Paulista de Artes, São Paulo. 2007.

TAYLOR, N. et al. Preference of growing pigs for illuminances. Applied Animal Behaviour Science, Elsevier, Amsterdam, v. 96, n. 1-2, p.19-31, Jan., 2006.

TRICKETT, S.L.; GUY, J.H.; EDWARDS, S.A. The role of novelty in environmental enrichment for the weaned pig. Applied Animal Behaviour Science, Elsevier, Amsterdam, v. 116, n. 1, p. 45-51, Jan., 2009.

TUYTTENS, F.A.M. et al. Synthetic lying mats may improve lyingcomfort of gestating sows. Applied Animal Behaviour Science, Elsevier, Amsterdam, v. 114, n. 1, p. 76-85, Nov., 2008.

VAN DE WEERD, H. A. et al. A systematic approach towards developing environmental enrichment for pigs. Applied Animal Behaviour Science, Elsevier, Amsterdam, v. 84, n. 2, p. 101-118, Nov., 2003.

VAN DE WEERD, H. A. et al. Effects of species-relevant environmental enrichment on the behaviour and productivity of finishing pigs. Applied Animal Behaviour Science, Elsevier, Amsterdam, v. 99, n. 3-4, p. 230-247, Sep., 2006.

VAN DE WEERD, H. A.; DAY, J.E.L. A review of environmental enrichment for pigs housed in intensive housing systems. Applied Animal Behaviour Science, Elsevier, Amsterdam,v. 116, n. 1, p. 1-20, Jan., 2009.

VAN WAGENBERG, A.V. et al. Effect of floor cooling on farrowingsow and litter performance: field experiment under Dutch conditions. Transactions of the American Society of Agricultural and Biological Engineers, St. Joseph, v. 49, n. 5, p. 1521-1527, 2006. 
WAGENBERG, A.V. et al. Effect of floor cooling on farrowingsow and litter performance: field experiment under dutch conditions. Transactions of the American Society of Agricultural and Biological Engineering, St. Josheph, v. 49, p. 1521-1527, 2006.

WARAN, N.K.; BROOM, D.M. The influence of a barrier on the behaviour and growth of early-weaned piglets. Animal Production, v. 56, n. 1, p. 115-119, Feb., 1993.

WELLS, D.L. Sensory stimulation as environmental enrichment for captive animals: A review. Applied Animal Behaviour Science, Elsevier, Amsterdam, v. 118, n. 1-2, p. 1-11, Apr., 2009.

YEATES, J.W.; MAIN, D.C.J. Assessment of positive welfare: a review. The Veterinary Journal, Elsevier, Amsterdam, v.175, p. 293-300, 2008.

ZWICKER, B. et al. Short- and long-term effects of eight enrichment materials on the behaviour of finishing pigs fed ad libitum or restrictively. Applied Animal Behaviour Science, Elsevier, Amsterdam, v. 144, n. 1-2, p. 31-38, Feb., 2013.

ZWIRTES, M. Comportamento de suínos em fase de creche submetidos a baias com diferentes disposições de brinquedos. 2013. 31p. Monografia (Curso de Graduação em Zootecnia) - Universidade Federal de Santa Maria, Palmeira das Missões, 2013. 